\title{
Plant Density and Herbicides Affect Cucumber Productivity
}

\author{
Jack E. Staub ${ }^{1}$ \\ Vegetable Crops Research, U.S. Department of Agriculture, Agricultural Research Service, \\ Horticulture Department, University of Wisconsin, Madison, WI 53706
}

Larry D. Knerr ${ }^{2}$ and Herbert J. Hopen ${ }^{3}$

Horticulture Department, University of Wisconsin, Madison, WI 53706

Additional index words. chloramben, naptalam, genotype, spacing, Cucumis sativus

\begin{abstract}
The growth and yield of three cucumber (Cucumis sativus L.) genotypes were evaluated in 1987 and 1988 using three planting arrangements and four weed control treatments. A monoecious, indeterminate, normal leaf cultivar, Calypso, a gynoecious, normal leaf size determinate $x$ monoecious determinate $F_{1}$ hybrid (UW 11234), and a gynoecious, determinate little leaf inbred line (WI 50476) were evaluated for fruit yield at a 30-cm equidistant $(109,000$ plants/ha), a $20-\mathrm{cm}$ equidistant $(242,000$ plants/ha), and a $43-\mathrm{cm}$ row $(272,000$ plants/ha) spacing. Weed control treatments consisted of an unweeded control, naptalam benzoic acid at $6.7 \mathrm{~kg}$ ae/ha, chloramben at $4.5 \mathrm{~kg}$ ae/ha, and the herbicides in combination. Although number and weight of fruit per hectare increased with increasing plant density, fruit weight per plant decreased with increasing density. Fruit harvested from plants in unweeded control plots were significantly smaller than those in herbicide-treated plots, and fruit yield of unweeded control plots was lower than in nonweedy plots in 1987. In 1987, plants in plots treated with naptalam produced smaller fruit than plants in plots treated with chloramben or chloramben plus naptalam. Although fruit yield per plant in 1987 was similar in chloramben plots with or without naptalam and generally higher than that of plots treated only with naptalam, fruit yields from naptalam-treated plants were highest in 1988. 'Calypso' usually produced more and larger fruit than the determinate genotypes. Based on comparisons of fruit per plant under comparable spacings, productivity of WI 5047G was often lower and its fruit were smaller than those of normal leaf genotypes. Plants of WI 5047G may not attain the source capacity needed for adequate fruit growth before the onset of anthesis, thus producing smaller fruit. Chemical names used: (2-[(1-naphtalenylamino)carbonyl)benzoic acid (naptalam); 3-amino-2,5dichlorobenzoic acid (chloramben).
\end{abstract}

The cost of harvesting has risen dramatically in certain areas where pickling cucumbers are produced. Therefore, it has become increasingly attractive for some growers and processors to use mechanized harvesting. About 50\% of Michigan and $25 \%$ of Wisconsin production areas are harvested mechanically, and the field area involved probably will increase in the future.

Pickling cucumber yield per unit area for once-over harvest is $\approx 50 \%$ that of multiple harvest. Consequently, optimal economic return on investment from a once-over, machine-harvest operation lies in judicious management of resources. Competitive yields for once-over mechanical harvest can be realized only when plant populations are increased several times over those typically used in hand-pick operations. Optimum population density of indeterminate cultivars used in the $1960 \mathrm{~s}$ was reported to be 24,700 to 49,400 plants/ha for hand harvest and 197,600 to 247,000 plants/ha for hand and mechanical harvest (Lorenz, 1969). Putnam (1963) evaluated populations ranging from 54,340 to 214,890 plants/ha and found that the highest yield and number of fruit per plant were from 30.5-cm spacings between plants regardless of the distance between rows. It was later determined that plant den-

Received for publication 1 Mar. 1991. Accepted for publication 10 Sept. 1991. Mention of a trade name, proprietary product, or specific equipment does not constitute a guarantee or warranty by the U.S. Dept. of Agriculture or the Univ. of Wisconsin and does not imply its approval to the exclusion of other products that may be suitable. The cost of publishing this paper was defrayed in part by the payment of page charges. Under postal regulations, this paper therefore must be hereby marked advertisement solely to indicate this fact.

${ }^{1}$ Research Horticulturist and Associate Professor.

${ }^{2}$ Former Graduate Research Assistant. Current address: Ferry Morse Seed Co., Naples, FL 33999.

${ }^{3}$ Professor. sity and arrangement (Morrison and Ries, 1967), and time of harvest (Miller and Hughes, 1969; Morrison and Ries, 1967) significantly affected crop yield and value.

Studies using indeterminate hybrids released in the late 1960s and early 1970 (Cantliffe and Phatak, 1975) suggested that plant populations of up to 850,000 plants/ha could be used in onceover mechanical harvest operations. Pickling cucumber yields can be increased substantially by planting $\geq 500,000$ plants/ha. Nevertheless, commercial pickling cucumber plant populations for once-over harvest typically average $\approx 150,000$ plants/ha. Growers have been reluctant to use high plant populations because yield has not always increased with increasing plant densities within the range of 100,000 to 250,000 plants/ha (Cantliffe and Phatak, 1975).

Optimization of plant architecture for mechanical harvest is essential. The dwarf or determinate (Prend and John, 1976) and little leaf (Bowers et al., 1981) characteristics have potential for increasing once-over harvest yield in pickling cucumber through concentrated fruit set at high plant density. Dwarf plants have fewer lateral branches and shorter lateral stems than their indeterminate counterparts (Sandhu et al., 1972). Growth of determinate cucumber plants terminates as a consequence of the conversion of the sympodial bud into floral tissue (Hutchins, 1940). However, the advantage of the little leaf type seems more related to its associated habit of multiple branching, which results in a dense canopy (Bowers et al., 1981).

Weed interference is a major factor that limits yield in commercial cucumber production. Average annual loss in value of the pickling cucumber crop in the United States due to weeds is $\$ 17,359,000$ (Weed Sci. Soc. of America, 1984). Inadequate weed control interferes with once-over mechanical harvesting since weed growth adversely affects the operation of mechanical harvesters and the close plant spacings required for these sys- 
terns render mechanical weed control impractical (O'Sullivan, 1980).

Herbicides currently registered for cucumber production include bensulide, chloramben, ethalfluralin, DCPA, glyphosate, naptalam, sethoxydim, and paraquat. Chloramben limits plant growth by inhibition of root development (Weed Sci. Soc. of America, 1989). Cucumber tolerance to chloramben is marginal (Cole and Jordan, 1974), thereby requiring the addition of activated charcoal in the seed furrow as a safening agent (Rhone Poulenc, 1990). Most growers are reluctant to use chloramben in this manner due to the added costs of machinery, time, and materials.

Naptalam is an amide herbicide whose mechanism of action involves the blocking of indoleacetic acid transport (Weed Sci. Soc. of America, 1989). When applied in conjunction with chloramben, naptalam has been shown to elicit a safening effect by affecting the translocation and metabolism of chloramben (Knerr et al., 1991). Under field conditions, optimal safening is obtained by applying chloramben 24 to $72 \mathrm{~h}$ after naptalam application (Knerr and Hopen, 1989).

Soil moisture increases the activity of both naptalam and chloramben (Cardenas and Santelman, 1966). The safening effect of naptalam is not achieved under low soil moisture conditions (La Bonte and Hopen, 1985). However, the immediate application of water after planting and spraying increases chloramben toxicity when applied alone or in mixtures with naptalam (Knerr, 1987).

The release of determinate inbreds and hybrids possessing unique vining habits for once-over harvest must be accompanied by information on cultural practices that maximize fruit yield and quality. Therefore, our research was designed to evaluate the effects of planting arrangement and chemical weed control on the growth and yield of an indeterminate and determinate hybrid with normal size leaves, and a determinate little leaf inbred line of cucumber.

\section{Materials and Methods}

Experiments were conducted at the Univ. of Wisconsin-Madison Agricultural Research Station at Hancock on a Plainfield loamy sand (Typic Udipsamment; mixed, mesic; pH 6.8; 0.8\% organic matter) in 1987 and 1988. The experiment design was a split-split-split plot consisting of 2 years, three planting densities, three genotypes, and four herbicide treatments arranged in a randomized complete-block design with three replications (nested in years). Whole plots were years, subplots herbicides, sub-subplots were planting densities, and sub-sub-subplots were genotypes.

Planting densities included 30-cm (109,000 plants/ha) equidistant (plants situated equal distances from each other in rows $(30 \mathrm{~cm})$ on the planting bed), 20-cm equidistant (242,000 plants/ ha), and 43-cm row (272,000 plants/ha; $7.5 \mathrm{~cm}$ within row) spacing. These spacings were chosen because reports of yield increases from plant populations between 100,000 to 250,000 plants/ha are inconsistent, and, initially, growers most likely will use determinate types at these spacings.

Three seeds were sown in a designated plant position and thinned to one plant at 7 days after emergence. Plots were 6.1 $\times 1.5 \mathrm{~m}$ (length/width) and contained four or five rows, depending on the planting density. Border rows separated each treatment bed. Genotypes included the monoecious, indeterminate normal leaf (area of first fully expanded leaf 80 to 100 $\mathrm{cm}^{2}$ ) hybrid ('Calypso'), a gynoecious, normal leaf determinate $\times$ monoecious determinate $F_{1}$ hybrid (UW 11234), and a little leaf (area of first fully expanded leaf 30 to $40 \mathrm{~cm}^{2}$ ) determinate inbred (USDA, WI 50476). Leaf areas were determined using a leaf area meter (Model LI-3100, LI-COR, Lincoln, Neb; unpublished data). Weed control treatments consisted of an unweeded control, naptalam at $6.7 \mathrm{~kg}$ ae/ha, chloramben at 4.5 $\mathrm{kg}$ ae/ha, and both herbicides in combination.

Commercial formulations of chloramben and naptalam were applied preemergence with a tractor-mounted sprayer that delivered 190 liters $\cdot \mathrm{ha}^{-1}$. Plots were irrigated with $13 \mathrm{~mm}$ of water via an overhead system $24 \mathrm{~h}$ (1987) or $1 \mathrm{~h}$ (1988) after the application of herbicides to move them into the seed zone. Plots were irrigated three times weekly, based on soil evapotranspiration rates (Jury and Tanner, 1975; Tanner and Jury, 1976).

The middle $4.9 \times 0.9 \mathrm{~m}\left(4.4 \mathrm{~m}^{2}\right)$ of each plot was harvested to minimize border effects. Fruit (all sizes) were harvested and weighed, allowing for $10 \%$ commercial oversize on a per-plot basis to simulate a once-over machine harvest. Stand and fruit counts and fruit weight were measured and used to calculate fruit weight per plant. Oversized and misshapen fruit were included in the analysis, and the smallest grade harvested was la $(\approx 19 \mathrm{~mm}$ in diameter $)$.

Cucumber plants were rated at 3 weeks (two to three trueleaf stage) after planting $(0=$ plant death, $3=$ moderate plant injury, and $5=$ no plant injury) to determine their response to herbicides. In 1988, weed control was rated visually 3 weeks after planting $(0 \%=$ no weed control and $100 \%=$ complete weed control). Weed control in untreated plots was the consequence of suppresion (interference) by the cucumber plants.

All traits were subjected to analysis of variance, and mean separations of main effects were performed based on WallerDuncan K-ratio test. F tests were used to determine significance of interaction terms.

\section{Results}

There were differences $(P=0.01)$ among years, genotypes, spacings, and herbicides for the variables evaluated (Table 1). Lower plant stands and yield (fruits per plant) in 1988, which was a drought year, contributed to the observed differences between years. Main effects of spacing, herbicide, and genotype were observed in fruit counts per plant in both years. In each year, plant spacing affected fruit count, and herbicide application affected fruit count and weight. The number and weight of fruit per plant varied among genotypes and the only consistent two-way interaction between the main factors was genotype $\mathrm{x}$ herbicide. Because differences between years resulted in complex second order interactions, interpretation of results was achieved by tabularizing mean values by year for spacing, genotype, and herbicide (Tables 2, 3, and 4).

1987 experiment. Plant stands among genotypes were similar within a spacing (Table 2). Ultimate plant densities for the 242,000 and 272,000 plants/ha treatments were similar for 'Calypso' and WI 5047G (Table 3). Total fruit count in 'Calypso' and WI 50476 plots were highest and equal at 242,000 and 272,000 plants/ha. In contrast, fruit count and weight per plant were highest at 109,000 plants/ha in all genotypes examined.

Although genotypes did not differ in number of fruit produced within a spacing (Table 2), total fruit count was higher in ' $\mathrm{Ca}$ lypso' and WI 50476 plots at 242,000 and 272,000 plants/ha than at 109,000 plants/ha (Table 3). Fruit count per plant for UW 11234 was lower than for other genotypes at the higher densities (Table 2). Although fruit count per plant of 'Calypso' and WI 5047G was similar at the highest densities, 'Calypso' produced more fruit per plant than either UW 11234 (determi- 
Table 1. Analysis of variance of yield responses of three cucumber genotypes as influenced by plant spacing and preemergence herbicide in 2 years.

\begin{tabular}{|c|c|c|c|c|c|c|c|c|c|}
\hline \multirow[b]{3}{*}{ Source } & \multirow[b]{3}{*}{$\mathrm{df}$} & \multicolumn{8}{|c|}{ Mean squares } \\
\hline & & \multicolumn{4}{|c|}{1987} & \multicolumn{4}{|c|}{1988} \\
\hline & & Stand & $\begin{array}{l}\text { Fruit } \\
\text { (no.) }\end{array}$ & $\begin{array}{l}\text { Fruit/ } \\
\text { plant } \\
\text { (no.) }\end{array}$ & $\begin{array}{c}\text { Fruit wt/ } \\
\text { plant } \\
(\mathrm{kg})\end{array}$ & Stand & $\begin{array}{l}\text { Fruit } \\
\text { (no.) }\end{array}$ & $\begin{array}{l}\text { Fruit/ } \\
\text { plant } \\
\text { (no.) }\end{array}$ & $\begin{array}{c}\text { Fruit wt/ } \\
\text { plant } \\
(\mathrm{kg}) \\
\end{array}$ \\
\hline Block (B) & 2 & $1472 * * *$ & $6095^{* * *}$ & $0.8^{\mathrm{NS}}$ & $451 * * *$ & $952^{\mathrm{NS}}$ & $1089^{*}$ & $0.01^{\mathrm{NS}}$ & $0.01^{\mathrm{NS}}$ \\
\hline Spacing (S) & 2 & $11510 * * *$ & $5476^{* * *}$ & $5.8^{* * *}$ & $313^{* * *}$ & $16127^{* * *}$ & $13593^{* * *}$ & $0.32^{*}$ & $0.01^{\mathrm{NS}}$ \\
\hline$B \times S$ & 4 & $202^{\mathrm{NS}}$ & $720^{\mathrm{NS}}$ & $0.6^{\mathrm{NS}}$ & $43^{\mathrm{NS}}$ & $279^{\text {NS }}$ & $169^{\text {NS }}$ & $0.04^{\mathrm{NS}}$ & $0.01^{\mathrm{NS}}$ \\
\hline Genotype (G) & 2 & $783^{*}$ & $4^{\mathrm{NS}}$ & $1.7^{* * *}$ & $740^{* * *}$ & $504^{N S}$ & $64^{\mathrm{NS}}$ & $0.30^{*}$ & $0.19^{* * *}$ \\
\hline $\mathrm{S} \times \mathrm{G}$ & 4 & $82^{\mathrm{NS}}$ & $13^{\mathrm{NS}}$ & $0.6^{\mathrm{NS}}$ & $19^{\mathrm{NS}}$ & $408^{N S}$ & $575^{\mathrm{NS}}$ & $0.10^{\mathrm{NS}}$ & $0.01^{\mathrm{NS}}$ \\
\hline$B \times G$ & 4 & $99^{\mathrm{NS}}$ & $562^{\mathrm{NS}}$ & $0.5^{\mathrm{NS}}$ & $164^{* * *}$ & $205^{N S}$ & $163^{N S}$ & $0.21^{\mathrm{NS}}$ & $0.01^{\mathrm{NS}}$ \\
\hline$B \times G(S)$ & 8 & $38^{\mathrm{NS}}$ & $125^{\mathrm{NS}}$ & $0.1^{\mathrm{NS}}$ & $29^{\text {NS }}$ & $279^{N S}$ & $409^{N S}$ & $0.04^{N S}$ & $0.01^{\mathrm{NS}}$ \\
\hline Herbicide $(\mathrm{H})$ & 3 & $1459 * * *$ & $2500^{* * *}$ & $6.2^{* * *}$ & $1105^{* * *}$ & $3858^{* * *}$ & $6388^{* * * *}$ & $1.10^{* * *}$ & $0.03^{* *}$ \\
\hline $\mathrm{B} \times \mathrm{H}$ & 6 & $140^{\text {NS }}$ & $510^{\mathrm{NS}}$ & $0.3^{\mathrm{NS}}$ & $39^{\mathrm{NS}}$ & $800^{\text {NS }}$ & $836^{\text {NS }}$ & $0.28^{\mathrm{NS}}$ & $0.01^{\mathrm{Ns}}$ \\
\hline $\mathrm{S} \times \mathrm{H}$ & 6 & $159^{\mathrm{NS}}$ & $467^{\mathrm{NS}}$ & $0.3^{\mathrm{NS}}$ & $79^{\mathrm{NS}}$ & $366^{\mathrm{NS}}$ & $719^{\mathrm{NS}}$ & $0.17^{\mathrm{Ns}}$ & $0.01^{\mathrm{NS}}$ \\
\hline $\mathrm{G} \times \mathrm{H}$ & 6 & $182^{\mathrm{NS}}$ & $1044^{* *}$ & $0.9^{*}$ & $183^{* * *}$ & $467^{\mathrm{NS}}$ & $869 *$ & $0.23^{*}$ & $0.02^{*}$ \\
\hline
\end{tabular}

Table 2. Yield response among three cucumber genotypes within three planting densities.

\begin{tabular}{|c|c|c|c|c|c|c|c|c|c|}
\hline \multirow[b]{2}{*}{$\begin{array}{l}\text { Plant } \\
\text { density } \\
(1000 s)\end{array}$} & \multirow[b]{2}{*}{ Genotype } & \multicolumn{4}{|c|}{1987} & \multicolumn{4}{|c|}{1988} \\
\hline & & Stand $^{z}$ & $\begin{array}{c}\text { Fruit/ } \\
\text { plot } \\
\text { (no.) }\end{array}$ & $\begin{array}{l}\text { Fruit/ } \\
\text { plant } \\
\text { (no.) } \\
\end{array}$ & $\begin{array}{c}\text { Fruit wt/ } \\
\text { plant } \\
(\mathrm{kg})\end{array}$ & Stand & $\begin{array}{l}\text { Fruit } \\
\text { (no.) }\end{array}$ & $\begin{array}{l}\text { Fruit/ } \\
\text { plant } \\
\text { (no.) } \\
\end{array}$ & $\begin{array}{c}\text { Fruit wt/ } \\
\text { plant } \\
(\mathrm{kg})\end{array}$ \\
\hline \multicolumn{10}{|l|}{109} \\
\hline & $\begin{array}{l}\text { Calypso } \\
\text { UW 11234 } \\
\text { WI 5047G }\end{array}$ & $\begin{array}{l}19.1 \mathrm{a}^{\mathrm{y}} \\
27.3 \mathrm{a} \\
24.5 \mathrm{a}\end{array}$ & $\begin{array}{l}43.3 \mathrm{a} \\
44.5 \mathrm{a} \\
42.6 \mathrm{a}\end{array}$ & $\begin{array}{l}2.50 \mathrm{a} \\
1.79 \mathrm{~b} \\
1.75 \mathrm{~b}\end{array}$ & $\begin{array}{l}1.00 \mathrm{a} \\
0.73 \mathrm{a} \\
0.38 \mathrm{~b}\end{array}$ & $\begin{array}{l}16.2 \mathrm{a} \\
20.3 \mathrm{a} \\
26.6 \mathrm{a}\end{array}$ & $\begin{array}{l}17.6 \mathrm{a} \\
13.8 \mathrm{a} \\
25.8 \mathrm{a}\end{array}$ & $\begin{array}{l}0.88 \mathrm{a} \\
0.62 \mathrm{a} \\
0.71 \mathrm{a}\end{array}$ & $\begin{array}{l}0.23 \mathrm{a} \\
0.16 \mathrm{ab} \\
0.08 \mathrm{~b}\end{array}$ \\
\hline \multicolumn{10}{|l|}{242} \\
\hline & Calypso & $44.8 \mathrm{a}$ & $61.0 \mathrm{a}$ & $1.46 \mathrm{a}$ & $0.55 \mathrm{a}$ & $34.2 \mathrm{a}$ & $32.8 \mathrm{a}$ & $0.81 \mathrm{a}$ & $0.18 \mathrm{a}$ \\
\hline & UW 11234 & $52.5 \mathrm{a}$ & $59.4 \mathrm{a}$ & $1.22 \mathrm{~b}$ & $0.45 \mathrm{a}$ & $43.6 \mathrm{a}$ & $32.8 \mathrm{a}$ & $0.72 \mathrm{a}$ & $0.15 \mathrm{ab}$ \\
\hline & WI $5047 \mathrm{G}$ & $49.6 \mathrm{a}$ & $66.9 \mathrm{a}$ & $1.39 \mathrm{a}$ & $0.31 \mathrm{~b}$ & $29.3 \mathrm{a}$ & $19.3 \mathrm{a}$ & $0.54 \mathrm{~b}$ & $0.05 \mathrm{~b}$ \\
\hline 212 & Calypso & $54.3 \mathrm{a}$ & $68.3 \mathrm{a}$ & $1.39 \mathrm{a}$ & $0.53 \mathrm{a}$ & $61.9 \mathrm{a}$ & $57.6 \mathrm{a}$ & $0.92 \mathrm{a}$ & $0.22 \mathrm{a}$ \\
\hline & UW 11234 & $66.0 \mathrm{a}$ & $67.6 \mathrm{a}$ & $1.05 \mathrm{~b}$ & $0.37 \mathrm{~b}$ & $68.5 \mathrm{a}$ & $56.7 \mathrm{a}$ & $0.80 \mathrm{a}$ & $0.15 \mathrm{ab}$ \\
\hline & WI $5047 \mathrm{G}$ & $54.2 \mathrm{a}$ & $63.9 \mathrm{a}$ & $1.42 \mathrm{a}$ & $0.31 \mathrm{~b}$ & $57.8 \mathrm{a}$ & $54.8 \mathrm{a}$ & $0.89 \mathrm{a}$ & $0.08 \mathrm{~b}$ \\
\hline
\end{tabular}

${ }^{\mathrm{z}}$ Mean no. plants $/ 4.4 \mathrm{~m}^{2}$ plot.

${ }^{\mathrm{y}}$ Mean separation within continuous columns at $P=0.05$ according to Waller-Duncan K-ratio test, $\mathrm{K}$ $=100$.

Table 3. Yield response among three cucumber genotypes across three planting densities.

\begin{tabular}{|c|c|c|c|c|c|c|c|c|c|}
\hline \multirow[b]{2}{*}{ Genotype } & \multirow[b]{2}{*}{$\begin{array}{c}\text { Plant } \\
\text { density } \\
(1000 s)\end{array}$} & \multicolumn{4}{|c|}{1987} & \multicolumn{4}{|c|}{1988} \\
\hline & & Stand $d^{x}$ & $\begin{array}{l}\text { Fruit/ } \\
\text { plot } \\
\text { (no.) }\end{array}$ & $\begin{array}{l}\text { Fruit/ } \\
\text { plant } \\
\text { (no.) }\end{array}$ & $\begin{array}{c}\text { Fruit wt/ } \\
\text { plant } \\
(\mathrm{kg})\end{array}$ & Stand & $\begin{array}{l}\text { Fruit } \\
\text { (no.) }\end{array}$ & $\begin{array}{l}\text { Fruit/ } \\
\text { plant } \\
\text { (no.) }\end{array}$ & $\begin{array}{c}\text { Fruit wt/ } \\
\text { plant } \\
(\mathrm{kg})\end{array}$ \\
\hline \multirow[t]{3}{*}{ Calypso } & 109 & $19.1 b^{y}$ & $43.3 \mathrm{~b}$ & $2.50 \mathrm{a}$ & $1.00 \mathrm{a}$ & $16.2 \mathrm{c}$ & $17.6 \mathrm{a}$ & $0.88 \mathrm{a}$ & $0.23 \mathrm{a}$ \\
\hline & 242 & $44.8 \mathrm{a}$ & $61.0 \mathrm{a}$ & $1.46 \mathrm{~b}$ & $0.55 \mathrm{~b}$ & $34.2 \mathrm{~b}$ & $32.8 \mathrm{~b}$ & $0.81 \mathrm{a}$ & $0.18 \mathrm{a}$ \\
\hline & 272 & $54.3 \mathrm{a}$ & $68.3 \mathrm{a}$ & $1.39 \mathrm{~b}$ & $0.53 \mathrm{~b}$ & $61.9 \mathrm{a}$ & $57.6 \mathrm{a}$ & 0.92 a & $0.22 \mathrm{a}$ \\
\hline \multirow[t]{3}{*}{ UW 11234} & 109 & $27.3 \mathrm{c}$ & $44.5 \mathrm{a}$ & $1.79 \mathrm{a}$ & $0.73 \mathrm{a}$ & $20.3 \mathrm{c}$ & $13.8 \mathrm{c}$ & $0.62 \mathrm{a}$ & $0.16 \mathrm{a}$ \\
\hline & 242 & $52.5 \mathrm{~b}$ & $59.4 \mathrm{a}$ & $1.22 \mathrm{~b}$ & $0.45 \mathrm{~b}$ & $43.6 \mathrm{~b}$ & $32.8 \mathrm{~b}$ & $0.72 \mathrm{a}$ & $0.15 \mathrm{a}$ \\
\hline & 272 & $66.0 \mathrm{a}$ & $67.6 \mathrm{a}$ & $1.05 \mathrm{~b}$ & $0.37 \mathrm{~b}$ & $68.5 \mathrm{a}$ & $56.7 \mathrm{a}$ & $0.80 \mathrm{a}$ & $0.15 \mathrm{a}$ \\
\hline \multirow[t]{3}{*}{ WI $5047 \mathrm{G}$} & 109 & $24.5 \mathrm{~b}$ & $42.6 \mathrm{~b}$ & $1.75 \mathrm{a}$ & $0.38 \mathrm{a}$ & $26.6 \mathrm{~b}$ & $25.8 \mathrm{~b}$ & $0.71 \mathrm{a}$ & $0.08 \mathrm{a}$ \\
\hline & 242 & $49.6 \mathrm{a}$ & $66.9 \mathrm{a}$ & $1.39 \mathrm{~b}$ & $0.31 \mathrm{a}$ & $29.3 \mathrm{~b}$ & $19.3 \mathrm{~b}$ & $0.54 \mathrm{a}$ & $0.05 \mathrm{a}$ \\
\hline & 272 & $54.2 \mathrm{a}$ & $63.9 \mathrm{a}$ & $1.42 \mathrm{~b}$ & $0.31 \mathrm{a}$ & $57.8 \mathrm{a}$ & $54.8 \mathrm{a}$ & $0.89 \mathrm{a}$ & $0.08 \mathrm{a}$ \\
\hline
\end{tabular}

${ }_{\mathrm{Z}}$ Mean no. plants $/ 4.4 \mathrm{~m}^{2}$ plot.

${ }^{\mathrm{y}}$ Mean separation within continuous columns at $P=0.05$ according to Waller-Duncan's K-ratio test, $\mathrm{K}$ $=100$.

nate, normal leaf) or WI 5047G (determinate, little leaf) at 109,000 plants/ha.

Fruit weight per plant from WI 50470 was consistently low when compared with the other genotypes at the same density (Table 2). At the highest density, the fruit weight per plant of UW 11234 and WI 50476 was similar, but lower than that of 
Table 4. Yield responses of three cucumber genotypes to preemergence herbicides.

\begin{tabular}{|c|c|c|c|c|c|c|c|c|c|}
\hline \multirow[b]{2}{*}{ Genotype } & \multirow[b]{2}{*}{ Herbicide ${ }^{7}$} & \multicolumn{4}{|c|}{1987} & \multicolumn{4}{|c|}{1988} \\
\hline & & Standy & $\begin{array}{c}\text { Fruit/ } \\
\text { plot } \\
\text { (no.) }\end{array}$ & $\begin{array}{l}\text { Fruit/ } \\
\text { plant } \\
\text { (no.) }\end{array}$ & $\begin{array}{c}\text { Fruit wt/ } \\
\text { plant } \\
(\mathrm{kg})\end{array}$ & Stand & $\begin{array}{l}\text { Fruit } \\
\text { (no.) }\end{array}$ & $\begin{array}{l}\text { Fruit/ } \\
\text { plant } \\
\text { (no.) }\end{array}$ & $\begin{array}{c}\text { Fruit wt/ } \\
\text { plant } \\
(\mathrm{kg}) \\
\end{array}$ \\
\hline \multirow[t]{4}{*}{ Calypso } & Control & $52.1 \mathrm{a}^{\mathrm{x}}$ & 54.7 a & $1.20 \mathrm{~b}$ & $0.31 \mathrm{c}$ & $48.0 \mathrm{a}$ & $41.3 \mathrm{~b}$ & $0.87 \mathrm{~b}$ & $0.19 a b$ \\
\hline & Naptalam & $47.2 \mathrm{ab}$ & $66.7 \mathrm{a}$ & $1.44 \mathrm{~b}$ & $0.53 \mathrm{bc}$ & $49.9 \mathrm{a}$ & $67.0 \mathrm{a}$ & $1.33 \mathrm{a}$ & $0.31 \mathrm{a}$ \\
\hline & Chloramben & $31.6 \mathrm{bc}$ & $53.7 \mathrm{a}$ & $1.94 \mathrm{ab}$ & $0.77 \mathrm{~b}$ & $21.9 \mathrm{~b}$ & $13.8 \mathrm{c}$ & $0.55 \mathrm{~b}$ & $0.17 \mathrm{~b}$ \\
\hline & Combination & $26.6 \mathrm{c}$ & $55.2 \mathrm{a}$ & $2.55 \mathrm{a}$ & $1.17 \mathrm{a}$ & $29.9 \mathrm{ab}$ & $21.8 \mathrm{bc}$ & $0.72 \mathrm{~b}$ & $0.17 \mathrm{~b}$ \\
\hline \multirow[t]{4}{*}{ UW 11234} & Control & $54.7 \mathrm{a}$ & $33.7 \mathrm{c}$ & $0.63 \mathrm{c}$ & $0.11 \mathrm{c}$ & $50.1 \mathrm{a}$ & $42.7 \mathrm{a}$ & $0.82 \mathrm{a}$ & $0.13 \mathrm{a}$ \\
\hline & Naptalam & $51.4 \mathrm{a}$ & $51.3 \mathrm{bc}$ & $1.11 \mathrm{bc}$ & $0.42 \mathrm{~b}$ & $51.1 \mathrm{a}$ & $42.0 \mathrm{a}$ & $0.76 \mathrm{a}$ & $0.17 \mathrm{a}$ \\
\hline & Chloramben & $44.8 \mathrm{a}$ & $77.3 \mathrm{a}$ & $2.03 \mathrm{a}$ & $0.84 \mathrm{a}$ & $43.4 \mathrm{a}$ & $28.7 \mathrm{a}$ & $0.60 \mathrm{a}$ & $0.14 \mathrm{a}$ \\
\hline & Combination & $43.4 \mathrm{a}$ & $66.3 \mathrm{ab}$ & $1.64 \mathrm{ab}$ & $0.68 \mathrm{a}$ & $31.8 \mathrm{a}$ & $24.3 \mathrm{a}$ & $0.66 \mathrm{a}$ & $0.16 \mathrm{a}$ \\
\hline \multirow[t]{4}{*}{ WI $5047 \mathrm{G}$} & Control & $49.8 \mathrm{a}$ & $43.8 \mathrm{a}$ & $0.97 \mathrm{~b}$ & $0.20 \mathrm{~b}$ & $49.8 \mathrm{a}$ & $30.9 a b$ & $0.65 \mathrm{ab}$ & $0.05 \mathrm{a}$ \\
\hline & Naptalam & $42.4 \mathrm{a}$ & $55.8 \mathrm{a}$ & $1.67 \mathrm{a}$ & $0.31 \mathrm{ab}$ & $51.1 \mathrm{a}$ & $54.2 \mathrm{a}$ & $0.94 \mathrm{a}$ & $0.10 \mathrm{a}$ \\
\hline & Chloramben & 39.7 a & $68.1 \mathrm{a}$ & $1.76 \mathrm{a}$ & $0.43 \mathrm{a}$ & $16.3 \mathrm{~b}$ & $15.4 \mathrm{~b}$ & $0.44 \mathrm{~b}$ & $0.05 a$ \\
\hline & Combination & $39.1 \mathrm{a}$ & $63.6 \mathrm{a}$ & $1.68 \mathrm{a}$ & $0.39 \mathrm{a}$ & $34.2 \mathrm{ab}$ & $32.7 \mathrm{ab}$ & $0.81 \mathrm{ab}$ & $0.10 \mathrm{a}$ \\
\hline
\end{tabular}

${ }^{\mathrm{z}}$ Preemergence herbicide rates; naptalam at $6.7 \mathrm{~kg} \cdot \mathrm{ha}^{-1}$, chloramben at $4.5 \mathrm{~kg} \cdot \mathrm{ha}^{-1}$.

${ }^{\mathrm{y}}$ Mean no. plants $/ 4.4 \mathrm{~m}^{2}$ plot.

${ }^{\mathrm{x}}$ Mean separation within continuous columns at $P=0.05$ according to Waller-Duncan's K-ratio test, $\mathrm{K}$ $=100$.

'Calypso'. The mean fruit weight per plant of 'Calypso' and UW 11234 was higher than that of WI 50470 at 109,000 plants/ ha, while fruit weight per plant of WI 50476 was similar at all spacings (Table 3). Although flowering time of WI 50476 was delayed ( $\approx 4$ to 5 days) when compared with the other genotypes tested, time to harvest (fruit maturity) was similar to that of the other genotypes used. Mature fruit weight ranged from 52 to $326 \mathrm{~g}$.

Plant stands in control plots within densities were similar to each other $(P=0.05)$. Herbicide treatment affected the stand of 'Calypso' plants (Tables 1 and 4). When compared with control or naptalam plots, plant stands in plots treated with a combination of naptalam and chloramben were low but not different than stands in plots where chloramben was applied alone (Table 4). Herbicide treatments did not affect fruit count per plant. In contrast, the fruit count in UW 11234 plots treated with chloramben alone or in combination with naptalam was higher than in the unweeded control and in plots where naptalam was applied alone. Regardless of genotype, fruit count and weight per plant were always higher in plots treated with chloramben plus naptalam than in the unweeded control. Moreover, no significant difference was detected in the number of fruit harvested per plant among plots where chloramben was applied alone or in combination with naptalam. 'Calypso' and UW 11234 fruits harvested from plots treated with a combination of chloramben and naptalam were heavier than plots treated with naptalam alone. Although the weight of 'Calypso' and WI 50476 fruit harvested from chloramben- and naptalam-treated plots was similar, UW 11234 fruit recovered from chloramben plots weighed more than those from naptalam plots.

1988 experiment. Plant stands among genotypes were similar within a spacing (Table 2), except for WI 50476, which had a lower stand than the other genotypes at 242,000 plants/ha. Except for WI 5047G, plant stands increased as densities increased. For all three cultivars, total fruit count per plot was directly correlated with plant count, since plant density did not affect fruit count per plant (Tables 2 and 3).

Genotypic differences were observed for fruit weight per plant within a spacing (Table 2). At each spacing, the fruit harvested from WI 50476 plants weighed less than 'Calypso' fruit but were similar in weight to UW 11234 fruit.
As in 1987, plant stands in control plots within subplots were similar $(P=0.05)$ and herbicide treatment affected stands of all genotypes, except UW 11234 (Tables 1 and 4). Plant stands in 'Calypso' and WI 50476 plots treated with chloramben were significantly lower than those in control plots. Plant stands in plots treated with naptalam alone or in combination with chloramben were similar and did not differ from stands in control plots.

More fruit were recovered from naptalam-treated plots than from chloramben plots. No differences in fruit count were detected between unweeded control plots and plots treated with a combination of chloramben and naptalam. On a per-plant basis, 'Calypso' treated with naptalam produced a higher fruit count and weight than when treated with other herbicides. In contrast, fruit yield of WI 5047G plants grown in plots treated with naptalam alone or in combination with chloramben was similar. Moreover, fruit weight per plant did not differ among herbicidetreated and unweeded control plots.

Mean phytotoxicity ratings of cucumber plants in control (5.0) and naptalam (4.7) treated plots were similar. However, plants in plots treated with chloramben alone (rated 2.7) or in combination with naptalam (rated 3.0) showed significant phytotoxicity. Significant differences $(P=0.05)$ in phytotoxicity ratings among genotypes were observed. WI 50476 was more sensitive (rated 3.6) to the effects of chloramben than UW 11234 (rated 4.0). The response of 'Calypso' plants (rated 3.9) was similar to that of the other two genotypes.

During both years, weeds present in the plots included yellow foxtail (Setaria lutescens L.), common lambsquarters (Chenopodium album L.), redroot pigweed (Amaranthus retroflexus L.), common ragweed (Ambrosia artemisiifolia L.), and carpetweed (Mollugo verticillata L.). Common lambsquarters and redroot pigweed were most frequent, but in no case did the weed population cover more than $\approx 25 \%$ of the area in treated plots. Chloramben alone or in combination with naptalam was equally effective in controlling weeds (97\% to $99 \%$ ) and better than naptalam alone $(57 \%)$.

\section{Discussion}

Development of dwarf or bush processing cucumber genotypes would be desirable if densely spaced plants allowed con- 
centrated fruit set. Concentrated fruit set might provide a yield advantage in once-over mechanical harvest operations. Further, if a crop could be produced in 39 to 45 days in some northern areas of the United States, a second crop might be grown. In this study, we evaluated two determinate plant types possessing different growth habits to measure yield at three densities and herbicide treatments.

Early in the development of mechanical harvesting, it was recognized that cultivars grown at high population densities must flower uniformly to realize optimum yields (Zahara and Sims, 1966). Genotype is important in determining sex expression under high plant densities (Nienhuis et al., 1984). For instance, hybrids made with determinate inbreds had more stable sex expression at high plant densities than indeterminate genotypes (Lower et al., 1983; Prend and John, 1976). Lower et al. (1983) compared indeterminate gynoecious inbreds and hybrids (gynoecious indeterminate $\mathrm{x}$ monoecious determinate or indeterminate) grown in various planting arrangements (single-row plots $84,000,128,000$, and 250,000 plants/ha; three-row plot 252,000 plants/ha), and found that plant density had little effect on the number of pistillate flowers in nonisogenic inbred lines. However, high density reduced pistillage flowers in indeterminate $\mathrm{x}$ indeterminate hybrids, while the indeterminate $\mathrm{x}$ determinate hybrid was unaffected. Likewise, the number of staminate flowers per plant on the gynoecious inbreds and indeterminate $\mathrm{x}$ determinate hybrids was small and was unaffected by density, while staminate flower production increased in indeterminate $\mathrm{x}$ indeterminate hybrids with increasing density. In a similar study, Nienhuis et al. (1984) evaluated two gynoecious inbreds and four hybrids (gynoecious indeterminate $\mathrm{x}$ monoecious determinate or indeterminate) at densities of 'one, two, or four plants per hill at populations of 84,000 plants/ha. When compared with indeterminate $\mathrm{x}$ indeterminate hybrids, indeterminate $\mathrm{x}$ determinate hybrids had a higher percentage of pistillate nodes per plant as the number of plants/hill was increased. This result indicates that the determinate gene enhanced pistillate expression.

In a study of yield from once-over harvest, dwarf gynoecious hybrids at 200,000 plants/ha averaged $19.0 \mathrm{Mg} \cdot \mathrm{ha}^{-1}$, compared with $7.0 \mathrm{Mg} \cdot \mathrm{ha}^{-1}$ for 'Pioneer', a predominately gynoecious hybrid (Prend and John, 1976). Based on data from small plots, projected yields for 'Calypso', the determinate gynoecious hybrid UW 11234, and the gynoecious determinate little leaf inbred WI 50470 at 242,000 plants/ha were 23.9, 23.8, and 17.2 $\mathrm{Mg} \cdot \mathrm{ha}^{-1}$, respectively, in 1987 and 7.7, 7.5, and $2.2 \mathrm{Mg} \cdot \mathrm{ha}^{-1}$, respectively, in 1988. The differences observed between years may be attributed in part to the drought conditions of 1988.

Using indeterminate 'Bounty' and 'Premier' grown at eight population densities ranging from 50,000 plants $(46 \times 46 \mathrm{~cm}$ spacing) to 850,000 plants/ha $(10 \times 10 \mathrm{~cm}$ spacing $)$ Cantliffe and Phatak (1975) determined that yield (value or weight) increased at high plant densities. Indeterminate and determinate plants arranged in 20 - and $30-\mathrm{cm}$ equidistant spacing resulted in an increase in total fruits per unit area at high plant density. Nevertheless, concomitant decrease in the number and weight of fruit per plant was recorded with increasing plant populations in all genotypes evaluated, which confirms results of Cantliffe and Phatak (1975). Although genotypes reacted similarly at certain spacings $(242,000$ plants/ha) genotypic differences were observed. 'Calypso' was more productive (fruits per plant) at 109,000 plants/ha when compared with UW 11234 or WI 50470 (Table 2; 1987). At 242,000 and 272,000 plants/ha, fruit of 'Calypso' were heavier (1987 and 1988) than fruit of WI 50470 and UW 11234, perhaps indicating that photosynthetic optimums (maximum net canopy assimilation rates) had been reached (Widders and Price, 1989) and that plants, under the competitive restrictions of this experiment, were producing smaller fruit as a direct result of source limitations.

Little leaf inbred WI $5047 \mathrm{G}$ performed poorly when compared to 'Calypso' and UW 11234 at the high densities evaluated. Vines of WI $50476(\approx 20$ to $30 \mathrm{~cm})$ were shorter than those of UW 11234 ( $\approx 60$ to $90 \mathrm{~cm})$. WI $5047 \mathrm{G}$ is an unbranching dwarf designed to produce one fruit per plant at high density. If this could be accomplished, then, theoretically, very high plant densities (500,000 to 850,000 plants/ha) could be attained, thereby increasing yield per hectare for once-over harvest. WI 50476 plants produced as many fruits as the other genotypes, but these fruit were small:

Widders and Price (1989) suggested that net photosynthetic capacity might limit fruit productive potential in pickling cucumbers. Pharr et al. (1985) indicated that fruit growth measured by weight gain was very rapid (3.3 g-h-l). Moreover, fruit growth was highly competitive with vegetative growth, and carbon assimilation was accelerated as cucumber leaves responded to strong sink demands imposed by growing fruit.

Our data for WI 5047G might have been expected, based on the work of Pharr et al. (1985) and Widders and Price (1989). At least two explanations might be given for the strong tendency of cucumber plants to grow a limited number of fruit simultaneously: 1) It may be a consequence of a strong internal competition, limiting fruit and vegetative development; or 2) small vine size may limit the rate of assimilate partitioning during reproductive growth, thus lowering the mean rate of expansive fruit growth. Plants of WI 50476, which are determinate and little leaf, may either not attain the source capacity needed for adequate fruit growth or not achieve adequate partitioning rates, thus producing smaller fruit. Further, although plants of WI 5047G appeared healthy, they matured (vegetatively) more slowly than the other strains evaluated (data not presented). This reduced rate of growth (rate and extent of canopy development) may have reduced its ability to compete with surrounding weed species, thus leading to low yields in control plots. The fact that WI 50476 performed comparatively better in plots treated with herbicide lends support to this hypothesis.

For some segments of the processing industry, the harvesting of small cucumbers $(<2.2 \mathrm{~cm}$ in diameter) would be attractive if a genotype could be identified that provided a significant yield advantage. At the row spacings used, fruit yields (fruit per plant) of WI 5047G were significantly higher (1987) than of UW 11234, especially in the smaller fruit sizes. If cultural practices were optimized for a short $(30 \mathrm{~cm})$ gynoecious determinate genotype such as WI 5047G, then perhaps the production of small fruits for pickling would be economically feasible. The introduction of a delayed flowering and multiple branching habit into such a genotype might allow for the production of a larger photosynthetic base before fruit set and might, therefore, alleviate source/sink limitations, if they do exist.

Chloramben metabolism in plants resulted in the formation of conjugates rather than degradation of the herbicide (Stoller and Wax, 1968). Adding naptalam to chloramben could result in a safening effect that potentially could allow the use of chloramben for chemical weed control in cucumber (Knerr and Hopen, 1989). From laboratory studies, the mode of safening action of naptalam on chloramben in cucumber appears, in part, to result from a reduction in the amount of free chloramben present in the leaves via an alteration of chloramben metabolism and a 
restriction of chloramben translocation to the leaves (Knerr et al. 1991). The safening effect was inconsistent under field conditions and depended on proper levels of moisture for uptake (Knerr and Hopen, 1989; La Bonte and Hopen, 1985). Irrigation immediately following herbicide application would be preferable when naptalam is applied, followed by a 24- to 48-h delay in chloramben application (Knerr and Hopen, 1989). This procedure could allow the safening niechanism of naptalam to become established in the germinating seed before chloramben application. We might have observed that effect if we had used a delayed chloramben treatment. Although the use of a delayed application of chloramben in conjunction with naptalam application at planting could be used as a means of safely controlling weeds in cucumber, chloramben is not now being manufactured.

Yield data suggest a much more severe effect from chloramben in 1988 compared with 1987 (Table 4). This difference may be explained by the differences in timing of initial irrigation to the plots or yield differences. In 1987, the plots were first irrigated $24 \mathrm{~h}$ after planting compared with immediately after planting in 1988. Therefore, in 1987 the seeds may have imbibed already present soil moisture for up to $24 \mathrm{~h}$ before the herbicides could reach them, effectively delaying chloramben uptake. In 1988, the herbicides were immediately moved into the seed zone, via overhead irrigation, thereby being immediately available for uptake. As chloramben is activated by soil moisture (Cardenas and Santelman, 1966), differing levels of moisture, which could affect activation and delivery of the herbicide to the seed zone, may explain differing levels of toxicity.

Light, water and/or nutrient availability can all become limiting at high plant densities, when plant interactions have a major effect on the growth and productivity of individual plants (Widders and Price, 1989). Water and mineral nutrients are not normally limiting factors during the early stages of plant competition under close spacing (O'Sullivan, 1980; Widders and Price, 1989). However, as a cucumber crop grown at a high plant population reaches anthesis, competition among neighboring plants (Widders and Price, 1989), for soil moisture (Cantliffe and Phatak, 1975; O'Sullivan, 1980; Tan et al., 1983) and N (O'Sullivan, 1980) becomes a significant growth-limiting factor. 'Calypso' performed better than the determinate lines in the unweeded control (statistics not presented), which suggests that 'Calypso' was more competitive with weed growth than the determinate genotypes used. This difference is, at least in part, due to the slow growth of the determinate genotypes, which were under strong weed interference in some cases (i.e., unweeded control).

It is generally accepted that crop plants are more productive under equidistant spacing arrangements when compared with row spacings at similar plant densities (Cantliffe and Phatak, 1975). Equidistant plant spacings and a common row spacing were used to achieve plant densities that are generally considered intermediate to theoretical upper density optimums for onceover mechanical harvest of cucumbers. Using current technology (vacuum planting), seeds could be sown at a $20-\mathrm{cm}$ equidistant spacing. No economic advantage for implementing such technology at this spacing was detected for the genotypes evaluated. In addition, our data suggest that the determinate cucumber genotypes used were sensitive to interplant competition and had differential reaction to the herbicides examined. Therefore, optimal cultural practices must be determined for genotypes with unique vining habits before their release.

\section{Literature Cited}

Bowers, J.L., M.J. Goode, and M.E. Peerson. 1981. Studies with little leaf cucumber. Proc. Annu. Meet. Arkansas State Hort. Soc. 102:19.

Cantliffe, D.J. and S.C. Phatak. 1975. Plant population studies with pickling cucumbers grown for once-over harvest. J. Amer. Soc. Hort. Sci. 100:464-466.

Cardenas, J. and P.W. Santelman. 1966. Influence of irrigation and formualtion on activity of NPA, Amiben, and DCPA. Weeds 14:309-312.

Cole, A.W. and T.N. Jordan. 1974. Seedling and yield response of cucumber to naptalam and chloramben. Weed Sci. 22:604-607.

Jury, W.A. and C.B. Tanner. 1975. The advection modification of the Priestly and Taylor evapotranspiration formula. Agron. J. 67:840-842.

Hutchins, A.E. 1940. Inheritance in the cucumber. J. Agr. Res. 60:117-128.

Knerr, L.D. 1987. Naptalam as a safener for cucumber (Cucumis sativus L.) against the phytotoxic effects of chloramben. MS Thesis, Univ. of Wisconsin-Madison. p. 62-70.

Knerr, L.D. and H.J. Hopen. 1989. Naptalam as a safener against the phytotoxic effects of chloramben in cucumber (Cucumis sativus L. 'Addis'). Weed Technol. 3:445-449.

Knerr, L.D., H.J. Hopen, and N.E. Balke. 1991. Effect of naptalam on chloramben toxicity, uptake, translocation, and metabolism in cucumber (Cucumis sativus L.). Weed Sci. 39:27-32.

La Bonte, D.R. and H.J. Hopen. 1985. Apparent safening of chloramben for cucumbers by combination with naptalam. Proc. North Central Weed Control Conf. 40:41.

Lorenz, O.A. 1969. The mechanized growing and harvesting of vegetable crops in the west. HortScience 4:238-239.

Lower, R.L., O.S. Smith, and A. Ghaderi. 1983. Effects of plant density, arrangement, and genotype on stability of sex expression in cucumber. HortScience 18(5):737-738.

Miller, C.H. and G.R. Hughes. 1969. Harvest indices for pickling cucumbers in once-over harvested systems. J. Amer. Soc. Hort. Sci. 94:485-487.

Morrison, F.D. and S.K. Ries. 1967. Cultural requirements for once-over mechanical harvest of cucumbers for pickling. Proc. Amer. Soc. Hort. Sci. 90:339-346.

Nienhuis, J., R.L. Lower, and C.H. Miller. 1984. Effects of genotype and within-row spacing on the stability of sex expression in cucumber. HortScience 19:273-274.

O'Sullivan, J. 1980. Irrigation, spacing, and nitrogen effects on yield and quality of pickling cucumbers grown for mechanical harvesting. Can. J. Plant Sci. 60:923-928.

Pharr, D.M., S.C. Huber, and H.N. Sox. 1985. Leaf carbohydrate status and enzymes of translocate synthesis in fruit and vegetative plants of (Cucumis sativus L.). Plant Physiol. 77:104-108.

Prend, J. and C.A. John. 1976. Improvement of pickling cucumber with the determinate (de) gene. HortScience 11:427-428.

Putnam, A.R. 1963. Horticultural aspects concerned with the production of pickling cucumbers for once-over harvest. MS Thesis, Michigan State Univ. p. 59.

Rhone-Poulenc. 1990. Product label guide. Research Triangle Park, N.C. Sandhu, M.S., H.C. Mohr, and D.K. Knavel. 1972. Comparison of a genetic dwarf and a normal vine cultivar of cucumber. HortScience 3:287.

Stoller, E.W. and L.M. Wax. 1968. Amiben metabolism and selectivity. Weed Sci. 16:283-288.

Tan, S.C., J.M. Fulton, and V.W. Nuttall. 1983. The influence of soil moisture stress and plant populations on the yield of pickling cucumbers. Scientia Hort. 21:217-224.

Tanner, C.B. and W.A. Jury. 1976. Estimating evaporation and transpiration from a row crop during incomplete cover. Agron. J. 68:239-243.

Weed Science Society of America. 1984. Crop losses due to weeds in Canada and the United States. Weed Sci. Soc. Amer., Champaign, Ill. p. 1-22.

Weed Science Society of America. 1989. Herbicide handbook of the Weed Society of America. Sixth ed. Weed Sci. Soc. Amer. Champaign, Ill. p. $51-52,192-193$.

Widders, I.E. and H.C. Price. 1989. Effects of plant density on growth and biomass partioning in pickling cucumbers. J. Amer. Soc. Hort. Sci. 11:751-755.

Zahara, M. and W.L. Sims. 1966. Once-over mechanical harvesting for cucumbers. Calif. Agr. 20:9-10. 